\title{
Photonic-Crystal Fibres with Suspended Core - Numerical Analyses
}

\author{
T. Pustelny* and M. Grabka \\ Department of Optoelectronics at Institute of Physics \\ Silesian University of Technology \\ Krzywoustego 2, 44-100 Gliwice, Poland
}

\begin{abstract}
The paper gives a short description of light guidance mechanisms of index-guided photonic-crystal fibres. The results of numerical analyses of photonic-crystal fibres with a suspended core are presented. Such fibres are planned to be applied for investigation of magneto-optic effects in gaseous media.

PACS numbers: 78.20.Ls, 07.07.Df, 42.79.Pw, 42.81.-i
\end{abstract}

\section{The photonic-crystal fibres}

In conventional optical fibres, electromagnetic modes are guided by a phenomenon of total internal reflection on a core-cladding border. The core refractive index is higher than the refractive index of a cladding which is achieved by adequate doping of a central part of the based material [1]. After over 20 years of intense researches on optical fibres, including special type optical fibres, it is currently apparent that transmission capabilities of conventional optical fibres reach their limit. Over the past few years, essential efforts have been directed toward elaboration of photonic-crystal fibres (PCF) [2]. The photonic-crystal fibres are new optical structures in which a refractive index varies periodically in the transverse plane with geometrical disturbances of the order of an optical wavelength. Morphological microstructure induces into the fibre core radically modification of optical properties of such fibre [3].

In general, two types of guiding mechanisms are distinguished in photonic-crystal fibres, which classify the photonic-crystal fibres as: index-guiding photonic-crystal fibres and the photonic band-gap fibres [2-4].

For index-guiding fibres a waveguide consists of a glass core and a cladding with an array of airholes. The optical modes are guided through the fibres in the core of a higher refractive index than an effective refractive index of the cladding. The index-guiding fibres are an excellent example of two-dimensional structures because their properties are invariable in the third dimension, i.e., along the length of a fibre. Qualitatively, propagation of modes in the index-guiding photonic-

${ }^{*}$ corresponding author; e-mail: tpustelny@polsl.pl 
-crystal fibre can be described by the effective index method. The cladding with holes is characterized by an effective refractive index $n_{\text {eff-clad }}$. At the same time, the core refractive index has a value equal to the refractive index of pure silica $n_{\text {Si-core}}$. It makes possible to analyse index-guiding photonic-crystal fibres as step-index fibres with refractive indices $n_{\mathrm{Si} \text {-core }}$ and $n_{\text {eff-clad }}$, respectively $[2,4]$.

To the contrary, photonic band-gap fibres have a hollow core most often surrounded by an airholed cladding. In such a case, refractive index of the core is lower than that of the cladding. Electromagnetic field, however, still propagates in the core of the fibre, which is assured by the so-called photonic band gap effect [4]. This effect prevents light from propagation in a cladding material, thus light needs to propagate inside the hollow core.

These two guiding mechanisms depending on the material properties of used glass and on their geometrical structures determine the enormous optical properties of the photonic-crystal fibres [2-4].

The photonic band gap fibres are not analysed in this paper.

\section{The index guiding photonic-crystal fibres with suspended core. Results of numerical analyses}

In the Laboratory of Optical Fibres Technology at the University of Lublin, Poland the optical fibres with a suspended core were fabricated. The fibre of this kind is presented in Fig. 1. Such fibre will be analysed numerically below.

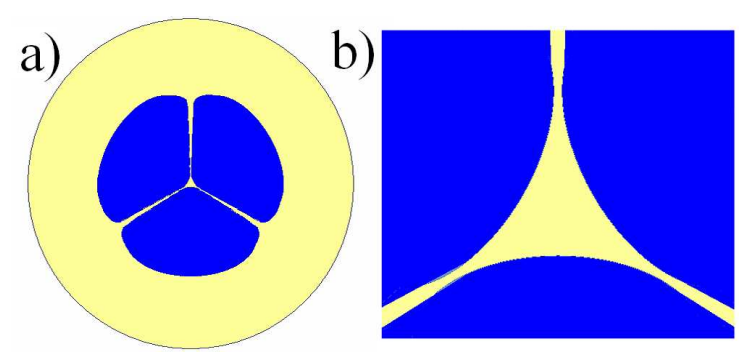

Fig. 1. The picture with a suspended core: (a) the fibre cross-section; (b) the suspended core (the fibre parameters: suspended-core dimension $-3 \div 4 \mu \mathrm{m}$; dimension of air holes $-50 \mu \mathrm{m} \times 35 \mu \mathrm{m}$; air bridge dimensions $-0.5 \div 3 \mu \mathrm{m}$; total diameter of fibre $-130 \mu \mathrm{m}$; core refractive index $n=1.444$ for $\lambda=1.55 \mu \mathrm{m})$.

The big air holes located inside these fibres enable insertion of gas into them. Introduction of the gases into the fibres, in addition to very high power density of light may lead to strong coupling between light and matter and observation of a number of interesting nonlinear optical phenomena such as electromagnetically induced transparency [5], slow light propagation [6, 7], nonlinear Faraday effects [8]. These effects can be applied in sensor technology, for example, for construction of ultraprecise magnetic field sensors. In a series of papers ultrahigh 
sensitive $\left(10^{-15} \mathrm{~T} / \mathrm{Hz}^{1 / 2}\right)$ techniques of very weak $\left(10^{-10}\right)[9]$ and stronger $\left(10^{-9}\right)$ relative sensitivity DC magnetic fields [10-12], as well as oscillating RF magnetic fields $[13,14]$ are presented. All these techniques are based on light-intensity dependent rotation of the polarisation plane of linearly polarised coherent light upon its propagation through a medium placed in external magnetic field, i.e. on nonlinear magneto-optical rotation [14]. Since strong light is necessary for observation of nonlinear magneto-optical rotation, it is usually analysed with intense light sources. However, the same effect could be observed with much weaker light sources in optical fibres, in which intensity of light propagating through a fibre core is very high $[11,14]$.

In this work we present an initial analysis of a possibility of application of suspended-core photonic-crystal fibres for observation of nonlinear magneto-optical effects. Numerical analysis of light propagation into the fibres was performed using the Optical Design Software System of OPTIWAVE SYSTEM Incorporation (Canada).

Figure 2 presents the relation of effective mode indices as a function of wavelengths $\lambda$ for the analysed fibres. The effective mode constants for TE and TM have nearly the same values and very strongly depend on light wavelengths.

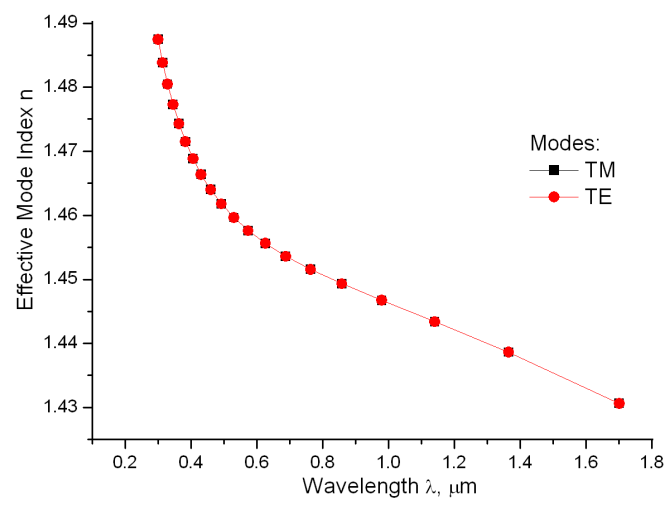

Fig. 2. The relation between effective mode index and light wavelength for the fibre with suspended core.

The distributions of the mode fields for the wavelength $\lambda=1.55 \mu \mathrm{m}$ inside the analysed fibre for TE and TM modes in Fig. 3 are presented. According to the results from Fig. 2, for both modes their field distributions are similar.

The obtained results show that in spite of such a small core of the fibres the entire energy of mode in the core is transmitted. The identical situations are observed also for wavelengths $\lambda<1.55 \mu \mathrm{m}$.

The presented results show that the possibilities of application of the fibres with suspended core for magneto-optical effect investigations in gases are very limited. Their application for nonlinear magneto-optical effects requires larger 

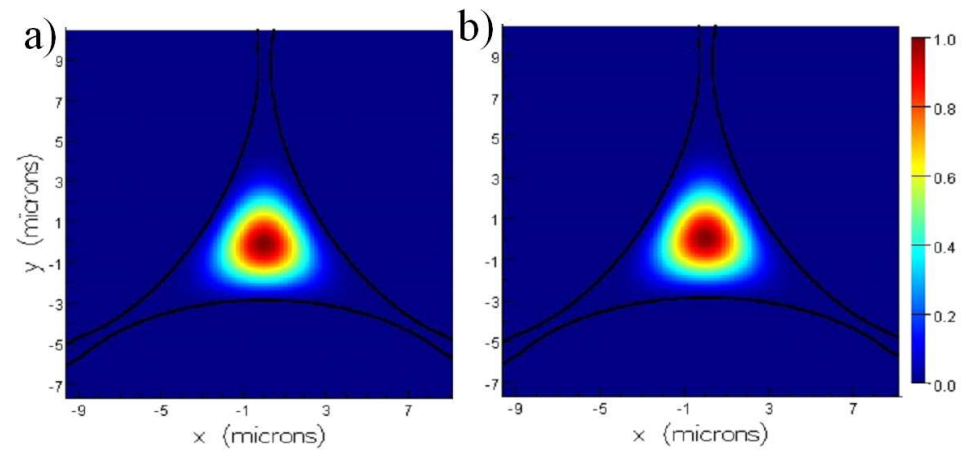

Fig. 3. The distribution of the mode fields for TE (a) and TM (b).
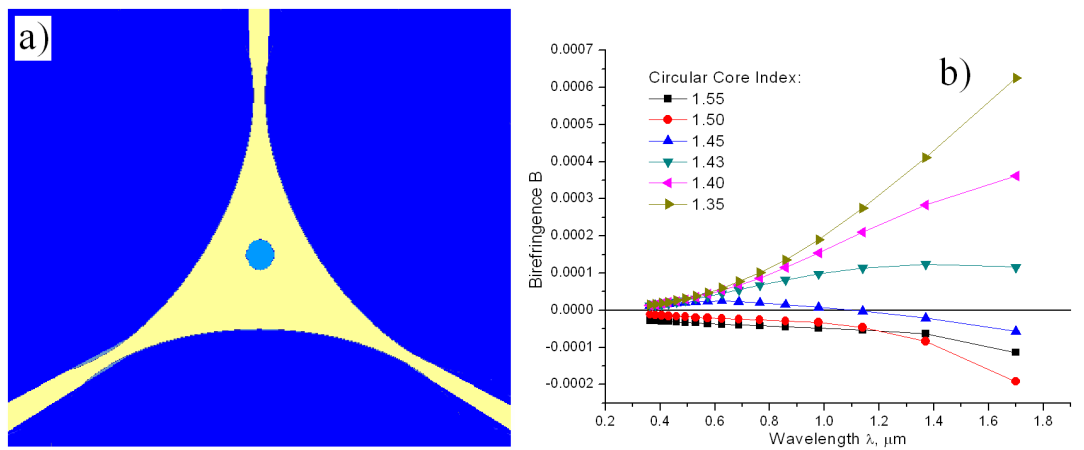

Fig. 4. (a) The optical fibre with suspended core modified by introduction in a central part of the core of a thin cylinder of different value refractive index; (b) the birefringence dependence on wavelength for various values of core refractive indices.
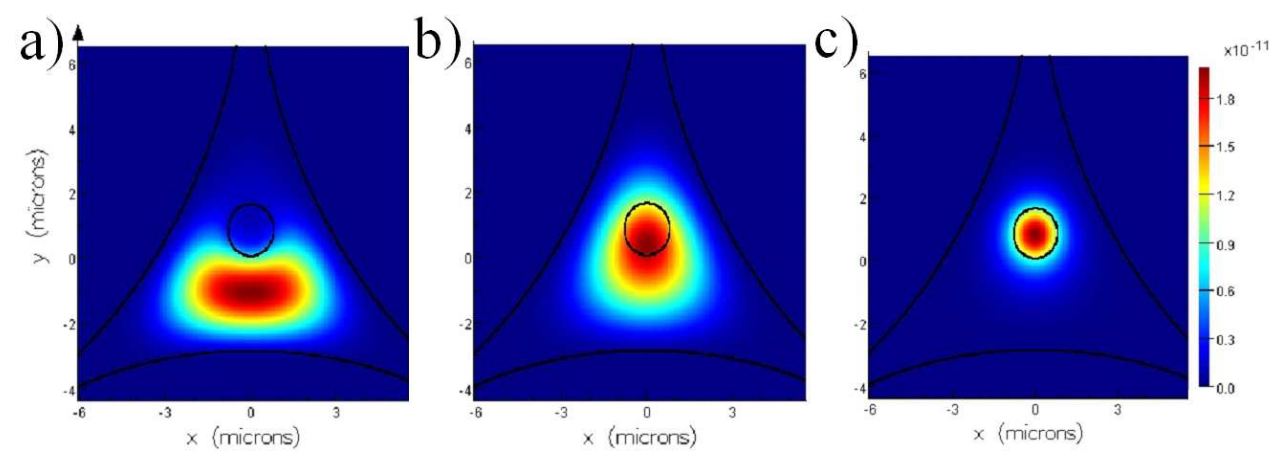

Fig. 5. The mode field distributions for the fibres with additional cylinder inside the suspended core depending on the refractive indices of cylinder $n_{\mathrm{cy}}$ : (a) $n_{\mathrm{cy}}=1.40$; (b) $n_{\text {cy }}=1.45 ;$ (c) $n_{\text {cy }}=1.50$.

overlap between light and airholes. Since light exists in the airholes in a form of evanescent wave, it implies fabrication of suspended-core with thinner core. 

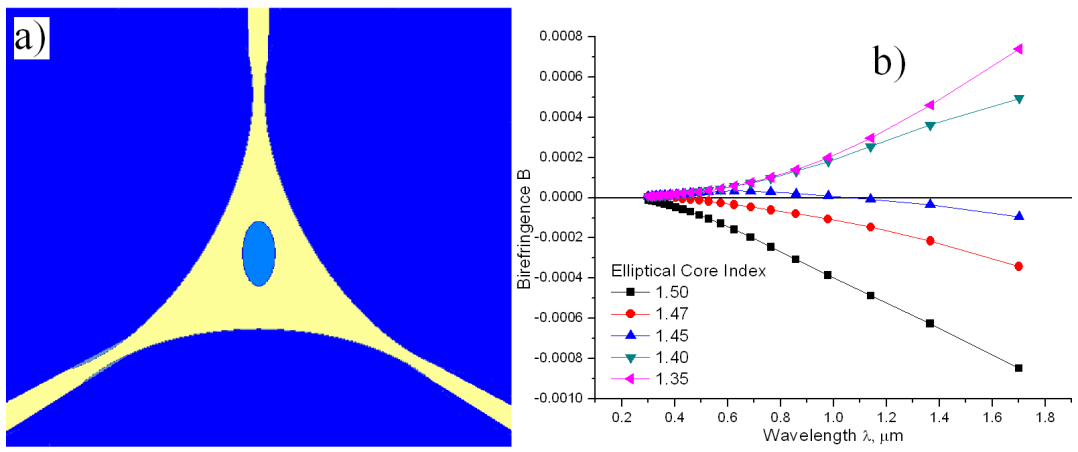

Fig. 6. (a) The optical fibre with suspended core with an additional elliptical core inside; (b) the birefringence dependence on wavelength for various values of core refractive indices.
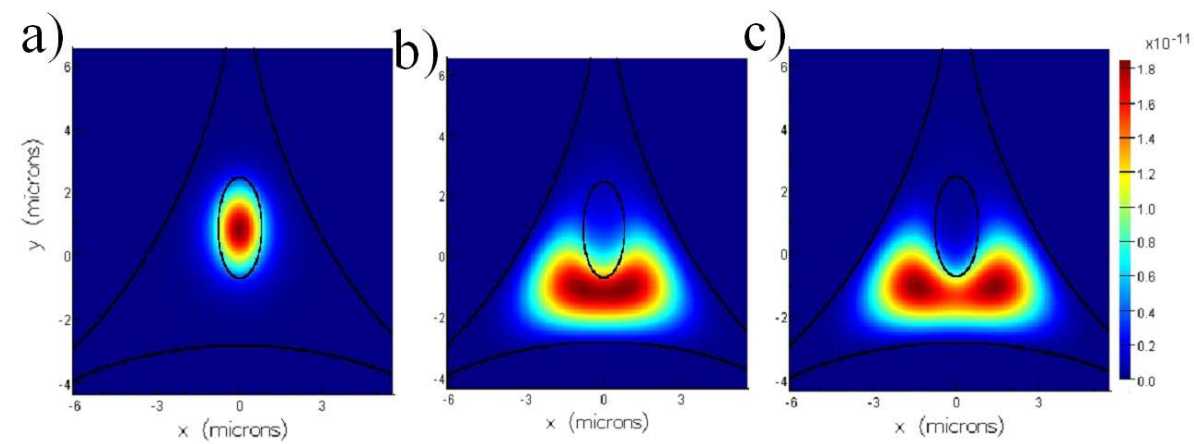

Fig. 7. The mode field distributions for the fibres with an additional elliptical core inside the suspended core depending on the refractive indices of cylinder $n_{\mathrm{cy}}$ : (a) $n_{\text {cy }}=1.40$; (b) $n_{\text {cy }}=1.45$; (c) $n_{\text {cy }}=1.50$.

In following analyses the suspended-core optical fibre was modified by introduction of a thin cylinder of a different value refractive index into a central part of the core. Figure 4 a presents the core structure with additional cylinder inside for its various refractive index values. Figure 4 shows spectral characteristics of birefringence [1] of the fibre for various refractive indices.

The distributions of the mode fields for optical fibres with an additional circular core inside the main core are presented in Fig. 5. In Figs 6 and 7 the optical properties of the photonic-crystal fibre with an additional elliptical core inside are shown.

\section{Conclusion}

The numerical analyses were carried out also for other configuration of cylindrical and elliptical additional cores placed inside the main suspended core not presented in this paper. In all analysed situations the energy was transmitted mainly 
inside the suspended cores penetrating to the air holes in very small quantity. The presented results of analyses seem to suggest that application of the photonic-crystal fibres with suspended core in investigations of quantum magneto-optic effects is not obvious and not easy problem. Our preliminary results (unpublished here) have been showing that the quantum magneto-optic effects have chances to be observed in the photonic-crystal fibres, in which the dimension of a suspended core is much smaller - a fraction of micrometer. (The results will be published in the nearest future.)

\section{Acknowledgments}

The work was sponsored by the State Committee for Scientific Research within the grant N N515 409534.

\section{References}

[1] T. Pustelny, Physical and Technical Aspects of Optoelectronic Sensors, SUT, Gliwice 2005 , p. 86.

[2] P. Russel, Science 299, 358 (2003).

[3] T.P. Hansen, J. Broeng, S.E.B. Libori, E. Knudsen, A. Bjarklev, J.R. Jensen, H. Simonsen, IEEE Photon. Technol. Lett. 13, 588 (2001).

[4] L. Xiao, W. Jin, M.S. Demojan, Opt. Express 15, 15637 (2007).

[5] E. Arimondo, in: Progress in Optics, Vol. XXXV, Ed. E. Wolf, Elsevier Sci. B.V., New York 1996, p. 259.

[6] D.F. Phillips, A. Fleischhauer, A. Mair, R.L. Walsworth, Phys. Rev. Lett. 86, $783(2001)$.

[7] C. Liu, Nature 409, 490 (2001).

[8] W. Gawlik, S. Pustelny, in: New Trends in Quantum Coherence and Nonlinear Optics, Ed. R. Drampyan, Nova Sci. Publ., New York 2009, p.41.

[9] D. Budker, D.F. Kimball, S.M. Rochester, V.V. Yashchuk, M. Zolotorev, Phys. Rev. A 62, 043403 (2000).

[10] S. Pustelny, A. Wojciechowski, M. Gring, M. Kotyrba, J. Zachorowski, W. Gawlik, J. Appl. Phys. 103, 063108 (2008).

[11] W. Gawlik, L. Krzemien, S. Pustelny, D. Sangla, J. Zachorowski, M. Graf, A.O. Sushkov, D. Budker, Appl. Phys. Lett. 88, 131108 (2006).

[12] K. Barczak, T. Pustelny, D. Dorosz, J. Dorosz, Europ. Phys. J. Special Topics 154, 11 (2008).

[13] M.P. Ledbetter, V.M. Acosta, S.M. Rochester, D. Budker, S. Pustelny, Phys. Rev. A 75, 023405 (2007).

[14] S. Pustelny, Ph.D. Thesis, Institute of Physics, UJ, Kraków, Poland, and University of California at Berkeley, USA, 2007. 